\title{
Performance of P-VCASS using a warning area based on pedestrian behavior
}

\author{
Tomotaka Wada ${ }^{1, \text { a) }}$ and Ruka Ohtani ${ }^{1}$ \\ ${ }^{1}$ Faculty of Engineering Science, Kansai University, \\ Yamate-cho 3-3-35, Suita, Osaka 564-8680, Japan \\ a)wadat@kansai-u.ac.jp
}

Abstract: In order for drivers to detect pedestrians at an early stage and prevent traffic accidents, we have been conducting research on P-VCASS (Pedestrian-Vehicle Collision Avoidance Support System). However, this system cannot adapt to cases where pedestrians suddenly run out to the road. In addition, since warnings are given only on the condition that the warning areas overlapped, there is a problem that warnings are given even when the risk of collision is low. To solve these problems, we propose a new method for determining the warning area based on pedestrian behavior. We show the effectiveness of the proposed method through experiments.

Keywords: P-VCASS, risk of collision, warning area, Wi-SUN Classification: ITS (intelligent transport system)

\section{References}

[1] Y. Kaneko and M. Hamaguchi, "Development of pedestrian to vehicle communication system," OKI Technical Review, 218, vol. 78, no. 1, pp. 72-77 2011.

[2] Y. Suzuki, E. Matsumoto, H. Shimada, and K. Sato, "Pedestrian safety support system based on pedestrian conditions of using the P2V communication," DICOMO2013 Symposium, pp. 717-722, July 2013.

[3] M. Bagheri, M. Siekkinen, and J.K. Nurminen, "Cellular-based vehicle to pedestrian (V2P) adaptive communication for collision avoidance," IEEE ICCVE, pp. 450-456, Nov. 2014. DOI: 10.1109/iccve.2014.7297588

[4] T. Wada, Y. Nakanishi, R. Yamaguchi, K. Fujimoto, and H. Okada, "Pedestrian oriented vehicular collision avoidance support system: P-VCASS," IEICE Trans. Fundamentals, vol. E93-A, no. 4, pp. 679-688, April 2010. DOI: 10.1587/ transfun.e93.a.679

[5] T. Wada, G. Nakagami, and S. Kawai, "New estimation method of pedestrian's running out into road by using pressure sensor and moving record for traffic safety," IEICE Trans. Fundamentals, vol. E100-A, no. 2, pp. 482-490, Feb. 2017. DOI: 10.1587/transfun.e100.a.482

[6] R. Otani, A. Shikishima, and T. Wada, "A study on vehicle-pedestrian communication system using warning ranges of mobile objects," The 34th International Conference on Information Networking (ICOIN 2020), pp. 517-522, Jan. 2020. DOI: $10.1109 /$ icoin48656.2020.9016444 


\section{Introduction}

Intelligent Transport Systems (ITS) has been developing to solve various problems in traffic environments. To prevent traffic accidents of pedestrians, there are various researches of pedestrian to vehicle communications $[1,2,3]$. We have been studying P-VCASS (Pedestrian-Vehicular Collision Avoidance Support System) [4, 5] for using GPS information. The objective of this system is to reduce accidents by warning and alerting the driver of the vehicle when the vehicle is about to collide with a pedestrian. However, the conventional P-VCASS system has prediction delay in pedestrian detection time when young people such as infants and elementary school children behave in an unexpected manner.

We propose a new method for determining the warning area in pedestrian-vehicle communication by solving the above problems and making the driver aware of highrisk pedestrians. The warning area is the area in which a pedestrian or a vehicle can move in a certain period. This is calculated from the speed and direction information included in the GPS information of the vehicle and the pedestrian. Each warning area uses a different form depending on the behavioral state of the pedestrian.

To verify the effectiveness of the proposed method, we carry out several experiments in actual road environment. We evaluate the reliability of the system based on warnings considering the presence or absence of warnings and the processing time.

\section{Conventional P-VCASS}

To avoid collision between vehicles and pedestrians, we have proposed a P-VCASS by estimating the moving state of pedestrians and changing a waring area dynamically according to the moving state of the vehicle [4].

However, it is difficult for P-VCASS to adapt to dangerous actions of pedestrians such as running out into road. To solve the problem, we have proposed a new estimation method of the pedestrian's action by using pressure sensor and moving record [5]. Through some experiments, we have confirmed that the proposed method is able to give waring to the driver one second earlier than the conventional method.

On the other hand, we have proposed a warning area determination algorithm using an accelerometer and a directional sensor [6]. By using this method, it is possible to warn the driver of the vehicle not only according to the relative distance between the vehicle and the pedestrian but also according to the condition of the pedestrian, so it is possible to prevent traffic accidents. However, since the warning is given only on the condition that the warning areas of the vehicle and the pedestrian overlap, there is a problem that the warning is given even when the risk of collision is low. In addition, since it is necessary for pedestrians to wear acceleration and orientation sensors, it is difficult to spread the system.

\section{Proposed method}

To solve the above problems, we propose a warning area calculation method that matches the danger of pedestrians by using only GPS. By judging the behavior of pedestrians and calculating the warning area, it is possible to make the driver recognize only more dangerous pedestrians. 


\subsection{Process of the proposed method}

The operation procedure of the proposed method is Step 1 to Step 5 below.

Step 1: Vehicles and pedestrian terminals each acquire position information from GPS.

Step 2: The pedestrian terminal periodically sends the acquired information to the neighboring vehicles.

Step 3: The vehicle determines the behavior of pedestrians based on the received information.

Step 4: The vehicle calculates the warning area of each vehicle and pedestrian.

Step 5: If the warning areas overlap, a warning is given to the driver.

In Step 3, the behavior of pedestrians is determined by the speed information and true direction information included in the GPS information. First, the pedestrian's traveling direction is classified into two categories, such as parallel or perpendicular to the vehicle's traveling direction. Next, it is classified into two categories of walking or stopping based on the speed information of pedestrians. After these classifications, if the direction of travel of the pedestrian is parallel to the vehicle or the pedestrian is stopping, the possibility of collision with the vehicle is low, so the risk of the pedestrian is set to be low. In other cases, the risk of pedestrians is set to be high.

In Step 4, the clothoid curve is used for the warning area of the vehicle when there is a high risk of pedestrians. A circle is used as the warning area for pedestrians. In a low risk of pedestrians, the waring area of vehicle and pedestrian is the rectangular and point, respectively.

In Step 5, a warning is given when the warning area of the vehicle and pedestrian calculated in Step 4 overlaps. At this time, a warning is given to the driver.

\subsection{Warning areas of vehicle and pedestrian}

We calculate the warning area of the vehicle from the GPS speed information. In addition, the warning area of the vehicle uses different shapes depending on whether the risk of pedestrians is high or low.

(1) High risk of pedestrians

In the proposed method, the clothoid curve is used for the warning area of the vehicle. The clothoid curve is a curve drawn by a vehicle when the steering wheel is turned at a constant angular velocity while keeping the vehicle speed constant. To draw this curve, three elements are required: radius of curvature $\mathrm{R}$, curve length $\mathrm{L}$, and clothoid parameter A. These three elements can be expressed by the following equation (1).

$$
\mathrm{R} \cdot \mathrm{L}=\mathrm{A}^{2}
$$

The curve length is calculated based on the vehicle speed. Figure 1 shows the warning area of the vehicle when there is a high risk to pedestrians.

(2) Low risk of pedestrians

If the risk of pedestrians is low, the rectangle is the warning area for the vehicle. The length of the short side of the rectangle is $1.5 \mathrm{~m}$ from the side of the vehicle. This is set as a scoring standard for proficiency tests at the Driver's License Examination Center, with the distance of 1 to $1.5 \mathrm{~m}$ or more from moving objects such as 


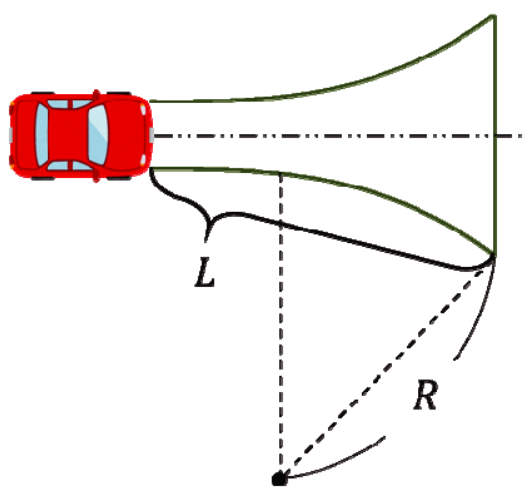

Fig. 1. Warning area of the vehicle

pedestrians, so $1.5 \mathrm{~m}$ with pedestrians. Also, the length of the long side should be the same as the warning area when there is a high risk of pedestrians.

The pedestrian warning area is calculated from the GPS speed information. If there is a high risk of pedestrians, the warning area is a circle with a radius of a [m] centered on the pedestrian position. The radius is the distance that can be moved in a certain period based on the speed of pedestrians. If the risk of pedestrians is low, the pedestrian position is used as the warning area.

\section{Performance evaluation by experiments}

In order to verify the effectiveness of the proposed method, we carry out experiments in two mobility models shown in Fig. 2. Model (a) is the situation two pedestrians are stopping near the pedestrian crossing. Model (b) is the situation two pedestrians are walking aross the road. The number of drivers of the vehicle is ten and the vehicle speed is $20 \mathrm{~km} / \mathrm{h}$. The wireless communication device is Wi-SUN (Wireless - Smart Utility Network) for long range communication. The communication interval is 0.5 seconds. The behavior of pedestrians and the warning area of each vehicle and pedestrian are determined, and then the system gives a cognitive warning to the driver of the vehicle when the warning areas overlap. The experiments are conducted 40 times in each model.

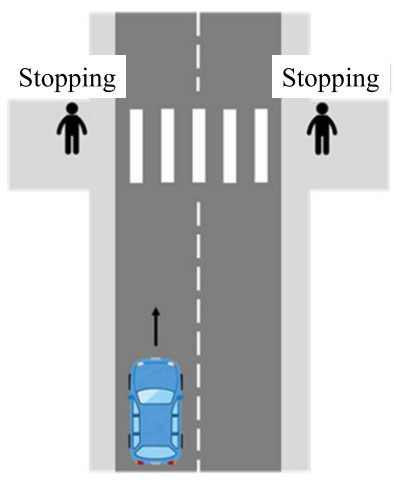

(a) Stopping

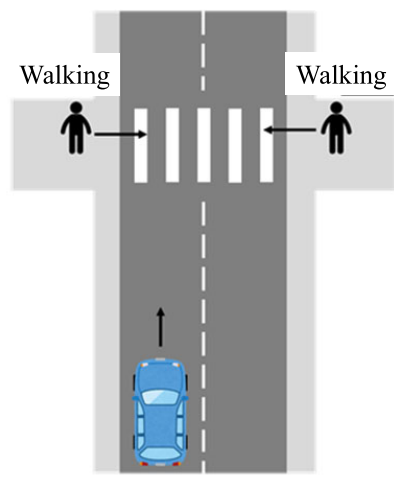

(b) Walking across the road

Fig. 2. Mobility models in the experiments

In the method of determining the warning area in pedestrian-vehicle communication, we verify whether a warning can be given at an appropriate timing when the 
warning areas overlap. TTC (Time To Collision) assumes to be 3 seconds. However, the warning is delayed because it takes time to acquire the GPS information and communicate between pedestrian and vehicle. The actual processing time takes at most one second in the experiments. So, the warning is judged by setting TTC to 4 seconds.

It is known that many people take a maximum of 1.5 seconds from the warning to the avoidance action such as stepping on the brake. Therefore, if the warning is given earlier than 1.5 seconds in consideration of the reaction time, it is an error warning.

We show the number of warnings in two mobility models in Table I. Pedestrian exists in each left and right beside the road 20 times. There is no error warning and the warning is given at a timing when collisions can be avoided sufficiently safely in the model (a). It can be seen that one error warning in actual state of no warning in the model (b). This was more than 1.5 seconds earlier warning due to the GPS information error. The correct warning rate is $97.5 \%$. We find that the proposed method can give a warning at an appropriate timing that can safely avoid collision.

Table I. The number of warnings in actual state
(a) Stopping
(b) Walking across the road

\begin{tabular}{|c|c|c|c|}
\hline \multicolumn{2}{|c|}{} & \multicolumn{2}{|c|}{ Decision } \\
\cline { 3 - 4 } & Warning & No warning \\
\hline \multirow{2}{*}{$\begin{array}{c}\text { Actual } \\
\text { state }\end{array}$} & Warning & 14 & 0 \\
\cline { 2 - 4 } & No warning & 0 & 26 \\
\hline
\end{tabular}

\begin{tabular}{|c|c|c|c|}
\hline \multicolumn{2}{|c|}{} & \multicolumn{2}{|c|}{ Decision } \\
\cline { 2 - 4 } & Warning & No warning \\
\hline \multirow{2}{*}{$\begin{array}{c}\text { Actual } \\
\text { state }\end{array}$} & Warning & 39 & 0 \\
\cline { 2 - 4 } & No warning & 1 & 0 \\
\hline
\end{tabular}

\section{Conclusions}

We have proposed a new method for determining the warning area based on pedestrian behavior in P-VCASS. We have shown the effectiveness of the proposed method through experiments. In a future work, we study a method of reducing the effect of GPS error, and investigate the performance in smaller transmission interval by the other communication media such as $5 \mathrm{G}$.

\section{Acknowledgments}

This research is partially supported by the Grant-in-Aid for Scientific Research (C) (No. 17K01309) from the Ministry of Education, Science, Sport and Culture of Japan. 